\title{
Template Assisted Deposition of Ferromagnetic Nanostructures: from Antidot Thin Films to Multisegmented Nanowires
}

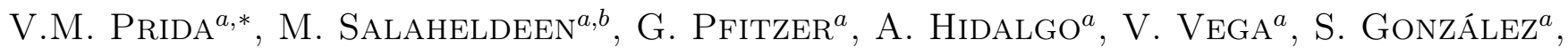 \\ J.M. TEIXEIrA ${ }^{a}$, A. Fernández ${ }^{a}$ AND B. HeRnANDO ${ }^{a}$ \\ ${ }^{a}$ Departamento de Física, Universidad de Oviedo, Calvo Sotelo s/n, 33007-Oviedo, Asturias, Spain \\ ${ }^{b}$ Physics Department, Faculty of Science, Sohag University, 82524-Sohag, Egypt
}

\begin{abstract}
The growth of nanostructured materials by means of different deposition methods employing nanoporous anodic aluminum oxide membranes as patterned templates has been widely used during last years due to the outstanding features displayed by these nanoporous templates. Here we report on the synthesis, morphology and magnetic properties exhibited by novel magnetic 1D and 2D nanostructured materials having nanowire or antidot thin films geometry, respectively, together to that of geometrically diameter modulated ferromagnetic nanowires. Their magnetic properties will be analyzed and discussed based on the different anisotropic behavior derived from their morphological and microstructural features.
\end{abstract}

DOI: 10.12693/APhysPolA.131.822

PACS/topics: 75.30.Gw, 75.70.Kw, 75.75.-c, 75.70.-i, 75.70.Rf

\section{Introduction}

Ordered arrays of nanostructured ferromagnetic materials have attracted huge interest in the research community due to their singular magnetic properties and potential applications they can exhibit in high-density recording media, magnetic sensors or energy conversion and microwave devices [1-3]. The development by Masuda and Fukuda of the two step anodization process for the fabrication of highly ordered nanoporous anodic aluminum oxide (AAO) templates [4] paved the way for the template-assisted deposition of a wide variety of nanomaterials. These nanomaterial families range from nanowire $[5,6]$ and nanotube arrays $[7]$ nanoparticle clusters $[8]$ and antidot thin films $[9,10]$, among others. The highly self-ordered hexagonal symmetry of the pores arrangement exhibited by the AAO templates displays narrow pore size distributions having well-defined interpore distances. Several deposition techniques, covering electrochemical deposition, chemical and physical vapor or solid deposition, spin-coating, dip-coating, sputtering, among others, have been successfully employed in combination with AAO templates, demonstrating the flexibility of this patterned material scaffold. Furthermore, advanced deposition techniques suitable for conformal coating of 3D structured substrates, such as atomic layer deposition (ALD), allow for additional control of the morphological parameters of AAO membranes, enabling independent control of pores diameter, while keeping constant the interpore distances [11]. In addition, advanced anodization protocols, including hard anodization [12,13], pulsed or sequential anodization [14], or lithography-guided anodization [15] allowed recently to obtain ideally ordered nanoporous templates, exhibiting also pore diameter

*corresponding author; e-mail: vmpp@uniovi.es modulations $[16,17]$. The spatial and periodic pores arrangement of the AAO templates, together with the confined growth of the magnetic material at the nanoscale deposited in the nanoporous alumina template, translate into the appearing of cooperative phenomena in the nanostructured materials, which in turn lead to tailor novel functional properties that differ from that of 3D bulk and $2 \mathrm{D}$ thin film systems $[18,19]$.

Here we report on more recent results obtained in our research group, related to the synthesis, morphology and magnetic properties exhibited by $1 \mathrm{D}$ geometrically diameter modulated ferromagnetic nanowires, together with novel magnetic 2D nanostructured materials with $\mathrm{Fe}$ antidots thin films geometry, respectively. Their magnetic properties will be analyzed and discussed based on the different anisotropic behavior derived from their morphological and microstructural features.

\section{Experimental procedure}

\subsection{Synthesis of nanoporous AAO templates}

Nanoporous AAO templates displaying highly ordered pore structures with narrow pore size distributions were obtained by electrochemical anodization of aluminum performed under mild anodic conditions. High purity aluminum foils were employed as starting substrates (Al 99.999\%, $0.5 \mathrm{~mm}$ in thickness, Goodfellow). The substrates, with typical size in the range of $2-3 \mathrm{~cm}^{2}$, are cleaned and then electropolished in perchloric acid and ethanol (25:75 vol.\%) mixture under an electropolishing voltage of $20 \mathrm{~V}$, applied between the samples (anode) and a platinum mesh counter-electrode. After obtaining a mirror-like surface finishing, the samples were cleaned under ultrapure water stream and dried in air. The conditions of further electrochemical anodization steps, carried out under the mild anodization regimes, determine ordering degree, size and surface distribution of the nanopores arrangement obtained in the AAO surface. 


\subsubsection{Mild anodization}

The two-step mild anodization approach, performed in $0.3 \mathrm{M}$ oxalic acid electrolytes at $1-3^{\circ} \mathrm{C}$ and $40 \mathrm{~V}$ of applied anodic voltage was employed to achieve highly ordered nanoporous alumina membranes with characteristic pore diameters of around $35 \mathrm{~nm}$, and center-to-center interpore distance of $105 \mathrm{~nm}$. A first anodization step of $24 \mathrm{~h}$ was carried out to achieve a highly self-ordered hexagonal pore distribution at the pores bottom [4]. Afterwards, the nanoporous AAO layer obtained during the first anodization step is removed in a selective chemical etching medium, consisting of an aqueous solution of $0.18 \mathrm{M} \mathrm{CrO}_{3}$ and $0.61 \mathrm{M} \mathrm{H}_{3} \mathrm{PO}_{4}$, at $30-40{ }^{\circ} \mathrm{C}$ during $12 \mathrm{~h}$. Finally, the second anodization step was performed under the same anodic conditions as the first one, and its time duration was controlled in order to adjust the total thickness of the highly ordered nanoporous AAO layer to the desired value, in the range from hundreds of nanometers to several tens of $\mu \mathrm{m}$. In addition, pore widening (PW) by means of isotropic chemical etching in $5 \% \mathrm{H}_{3} \mathrm{PO}_{4}$ acid solution at $30^{\circ} \mathrm{C}$ was employed by varying the etching time in the range of $0-35 \mathrm{~min}$, in order to increase the initial pore size from $35 \mathrm{~nm}$ up to $70 \mathrm{~nm}$, when desired. Mild-anodized nanoporous AAO membranes, obtained according the above explained procedure, have been employed within this work as templates for the fabrication of antidot thin films and diameter modulated nanowires, as it will be explained in the following sections.

Previously to the growing of magnetic nanowire arrays by electrochemical deposition in the pores of AAO templates, the remaining $\mathrm{Al}$ from the substrate was selectively dissolved by selective chemical etching in a solution of $3.6 \mathrm{~g} \mathrm{CuCl}_{2}$ and $50 \mathrm{ml}$ of $\mathrm{HCl}$ (37\% purity) in $100 \mathrm{ml}$ of ultrapure water. The alumina barrier layer, which occludes the pores bottom, was removed by exposing it to $5 \mathrm{wt} \% \mathrm{H}_{3} \mathrm{PO}_{4}$ acid solution at room temperature (RT) during $100 \mathrm{~min}$. Gold nanocontacts were deposited in the top or bottom-side of AAO templates by $\mathrm{Au}$ sputtering and further gold electrodeposition from a commercial gold plating solution (Orosene 999). This gold nanocontacts layer will serve as working electrode in the subsequent electrochemical deposition process.

\subsubsection{Surface functionalization of AAO templates by ALD}

The surface of AAO templates was functionalized by ALD before deposition of the gold nanocontacts and nanowires to protect them from corrosion. For that goal, the AAO membranes were coated with a protective $\mathrm{SiO}_{2}$ conformal layer of 2-5 $\mathrm{nm}$ in thickness, deposited by ALD on the alumina surface and previously to the electrodeposition of metallic nanowires, at a reactor temperature between $150{ }^{\circ} \mathrm{C}$ and $180^{\circ} \mathrm{C}$ from aminopropyltriethoxysilane $\left(100^{\circ} \mathrm{C}\right)$, water $(\mathrm{RT})$, and ozone (RT) that were employed as precursors and oxidant agents, respectively. Each cycle settings consisted of silane pulse times between 1 and $5 \mathrm{~s}$ and silane exposure times between 7 and $30 \mathrm{~s}[20]$.

\subsection{Diameter modulated nanowire arrays}

In order to develop nanowire arrays displaying modulations in their diameter and along their length it is necessary to produce engineered AAO templates displaying $3 \mathrm{D}$ nanometric features, such as nanopore diameter modulations along their thickness. For that purpose, a sequential approach that combines anodization, pore enlargement by isotropic chemical etching of PW steps and ALD functionalization, was employed [21]. Two samples (G1 and G2) have been prepared by following this process. The different steps are displayed in the scheme of Fig. 1.
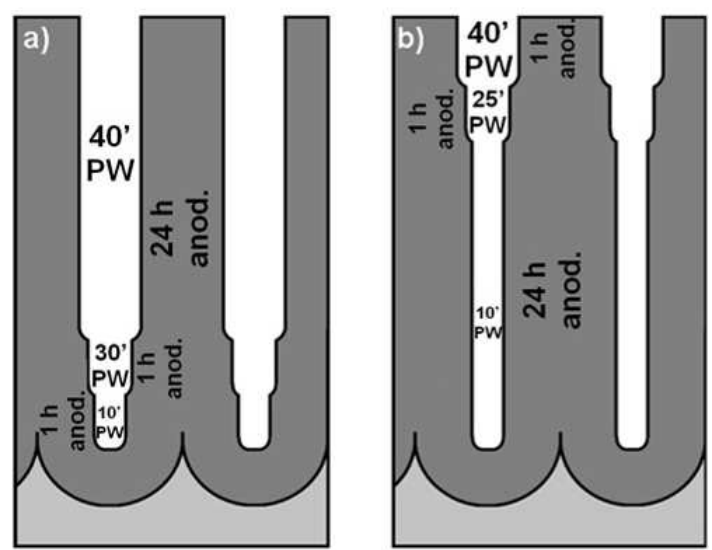

Fig. 1. Schematic drawing of the different steps followed during the synthesis of AAO nanoporous templates with diameter modulated nanopores: (a) sample G1, (b) sample G2.

In the case of G1 sample (Fig. 1a), a long time second anodization step of $24 \mathrm{~h}$ was performed under $0.3 \mathrm{M}$ oxalic acid electrolyte, as explained in Sect. 2.1.1. Afterwards, the sample was subjected to a PW step, by immersing it in $5 \% \mathrm{H}_{3} \mathrm{PO}_{4}$ acid at $30^{\circ} \mathrm{C}$ during $15 \mathrm{~min}$. Then, the sample was anodized for $1 \mathrm{~h}$ and the pores were widened for another $15 \mathrm{~min}$ under the same conditions as in the previous PW step. Finally, the last anodization was performed for $1 \mathrm{~h}$, followed by $10 \mathrm{~min}$ of PW. Therefore, the first long segment of the nanopores has an accumulated PW time of $40 \mathrm{~min}$, the intermediate one $30 \mathrm{~min}$ and the last segment only $10 \mathrm{~min}$ of PW, thus resulting in a differentiated pore enlargement with three sections. For sample G2 a similar approach was employed, but in an inverted geometry, with a second and third short anodization steps ( $1 \mathrm{~h}$ each) and a fourth anodization of $24 \mathrm{~h}$, resulting in three diameters modulated geometry, as depicted in Fig. 1b.

Afterwards, the open surface area of the nanoporous AAO membrane was coated with a thin $\mathrm{SiO}_{2}$ layer by performing 80 cycles of ALD deposition. This cover layer serves as passivation barrier that protects the nanowires surface against chemical etching. The remaining $\mathrm{Al}$ substrate and alumina barrier layer at the pores bottom were removed by chemical etching, as indicated previously, and gold nanocontacts are deposited in the top 
surface of the AAO membrane. These 3D nanostructured AAO membranes are then used as templates for the fabrication of diameter modulated Ni nanowire arrays.

Electrochemical deposition of $\mathrm{Ni}$ was performed at $1.2 \mathrm{~V}$ vs. $\mathrm{Ag} / \mathrm{AgCl}$ reference electrode, employing a Watts type electrochemical bath, which contains boric acid, nickel sulphate, and nickel chloride salts, with a $\mathrm{pH}$ of around 4, adjusted by adding several drops of $\mathrm{NaOH}$. The temperature of the Ni electrolyte was adjusted to $35^{\circ} \mathrm{C}$ prior to electrodeposition. For comparison, non-modulated channels of the nanoporous alumina membranes, with uniform nanopores diameter similar to both, the larger and smaller segments in samples G1 and G2 have been synthesized and filled with $\mathrm{Ni}$ nanowires. These samples were labeled as G3 and G4 (for small and large nanopores diameter, respectively). Microstructural analysis was performed by transmission electron microscopy (TEM, JEOL-JEM-2100). The magnetic properties of diameter modulated and non-modulated Ni nanowire arrays have been studied by hysteresis loops measured in a vibrating sample magnetometer (VSM Versalab, Quantum Design) at RT, by applying a magnetic field up to $\pm 3 \mathrm{~T}$, along both parallel and perpendicular directions to the length axis of the nanowires.

\subsection{Fe thin films and antidot arrays}

Continuous thin films of Fe, together with hexagonally ordered arrays of Fe antidots thin films, have been prepared by employing a vacuum thermal evaporation technique, which consists in the evaporation of the magnetic metal either on flat substrates or on the top surface of nanoporous anodic alumina membranes used as templates, respectively [22]. Starting nanoporous AAO membranes were fabricated by following the similar two-step anodization process explained above in Sect. 2.1.1, and followed by a $30 \mathrm{~min}$ PW step. The deposition of the thin films of $\mathrm{Fe}$ antidots arrays was carried out at RT $\left(\approx 20^{\circ} \mathrm{C}\right)$ on the top-surface of the nanoporous AAO serving as templates. The deposition process was performed in a high-vacuum system $\left(2.3 \times 10^{-6} \mathrm{mbar}\right)$, with base pressure $1.2 \times 10^{-6}$ mbar. The system employs a diffusion pump backed by rotary pumping together with a liquid nitrogen trap. A radiant heater, coupled to a temperature controller, was employed in order to keep the substrate temperature in the range of $20 \pm 2{ }^{\circ} \mathrm{C}$. The distance between the target and the substrate were $18 \mathrm{~cm}$, in order to get a low and constant deposition rate of around $1.0 \AA \mathrm{s}^{-1}$. The thickness of Fe thin films was controlled by using a quartz crystal microbalance for monitoring the layer thickness growth. Morphological properties of samples have been investigated by using a scanning electron microscope (SEM, JEOL 6100) equipped with an energy dispersive X-ray microanalysis system (EDX, Inca Energy 200). The geometric parameters of the Fe antidots thin films consist of holes with a diameter around of $60 \mathrm{~nm}$, interspacing distance between holes about $105 \mathrm{~nm}$ and film thickness ranging from $30 \mathrm{~nm}$ up to $100 \mathrm{~nm}$. The so obtained magnetic thin films also retain the high quality hexagonal ordered structure of nanoholes by repli- cating the nanoporous alumina template. The magnetic properties of non-patterned Fe thin films and the antidot arrays have been determined by performing hysteresis loops at RT $(300 \mathrm{~K})$ with a magneto-optic Kerr effect (MOKE) unit (NanoMOKE-3, Durham Magneto-optics). The magnetic field was applied along the in-plane and perpendicular to the plane (out-of-plane) directions of Fe antidots arrays and continuous thin films.

\section{Results and discussion}

\subsection{Fe antidots and thin film characterization}

Figure 2 displays the top-view surface by SEM of hexagonally ordered structure of nanoholes for the Fe antidot thin film, which are reproducing the spatial arrangement of the nanopores in the AAO template on which the $\mathrm{Fe}$ thin layer was deposited.

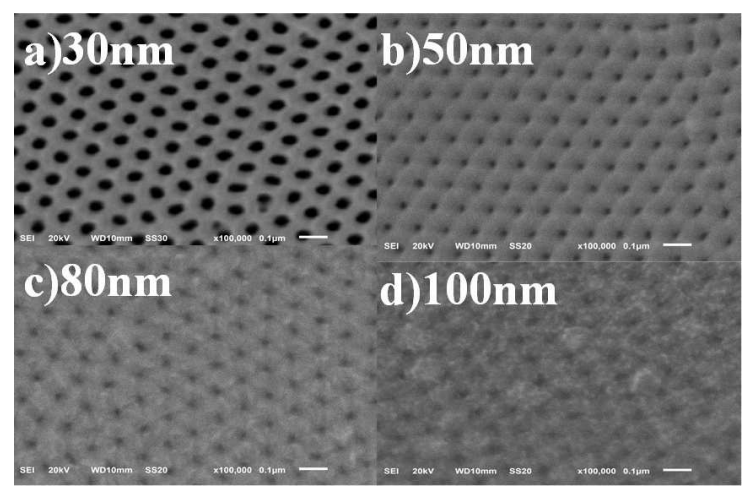

Fig. 2. SEM top-view surface of Fe antidot thin films with thicknesses ranging from $30 \mathrm{~nm}$ up to $100 \mathrm{~nm}$. Scale-bars indicate $100 \mathrm{~nm}$.

It can be observed that an increase in the Fe layer thickness leads to a decrease in the hole size of the antidots thin film, yielding a nearly occluded patterned structure for layer thicknesses higher than $100 \mathrm{~nm}$. This means a surface similar to the continuous thin film, as it will be also discussed below.

\subsection{Magnetic properties of Fe thin films and antidot arrays}

The hysteresis loop characteristics of the specimens have been investigated by MOKE magnetometer, measured along the in-plane $(\|)$ and out-of plane $(\perp)$ applied field directions with regard to both, the Fe continuous thin films and the antidots arrays. The azimuthal (inplane) and polar angular dependences of the hysteresis loops have been also performed to identify the easy magnetization axis.

Although the continuous thin films have a homogeneous composition of magnetic material, we have found that for all samples studied, the coercive field $\left(H_{c}\right)$ along the easy magnetization axis increases monotonously with the film thickness, $t$, as it can be shown in Fig. 3. The coercivity dependence on the thickness for the continuous thin films can be ascribed to the shape anisotropy and inhomogeneous magnetization rotation caused by the nanoscale roughness ascribed to thin film thickness increase. 
The thickness dependent roughness is strongly related to the film growth modes and the type of material being deposited. For the same film thickness, the rougher the surface the larger is the coercivity [23]. Consequently, by increasing the film thickness, $H_{c}$ rises due to the surface roughness and grain size increase, which results in an increase of the magnetic shape anisotropy for these thin films [24-26]. This observation agrees well with our experimental data, as shown in Fig. 3. The thickness of the Fe continuous thin film increases from 30 up to $100 \mathrm{~nm}$, giving rise to $H_{c}$ values between 101 and 160 Oe, respectively. Therefore, the magnetic behavior is dominated by the layer thickness of the Fe thin films, where the larger coercivity is obtained for the thin film with the higher thickness.

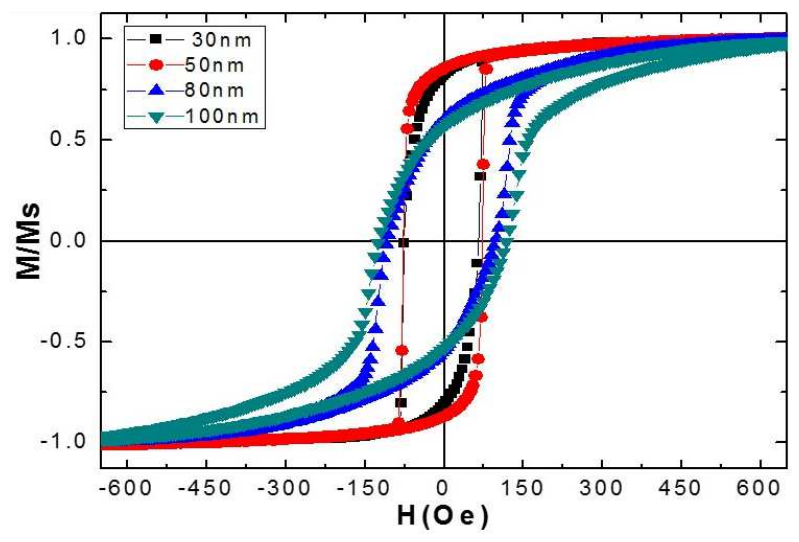

Fig. 3. Longitudinal Kerr hysteresis loops for Fe continuous thin films with different thicknesses $(30,50,80$, and $100 \mathrm{~nm})$.

The nanostructuring significantly affects the shape of hysteresis loops and drastically changes the magnetic behavior. As it can be seen in Fig. 4, a magnetic anisotropy with in-plane easy magnetization direction is derived for Fe antidots arrays due to the nanoholes induce a strong local shape anisotropy that tends to align the magnetization parallel to their edges. The intrinsic nature of such in-plane magnetic anisotropy is due to the shape of the specimens. Consequently, in-plane magnetization process is due to domain wall displacements giving rise to hysteresis loops with high squareness $(S)$ ratio, while magnetization rotation processes are responsible for the out-of-plane magnetization reversal direction.

By direct comparison between Fig. 3 and Fig. 4, corresponding to samples of continuous thin films and the thin films of antidot arrays, respectively, higher values of $H_{c}$ are found in the Fe antidots than the corresponding ones for the continuous films with the same layer thickness, which can be associated to an additional pinning effect originated by nanoholes. This pinning effect induced by nanoholes is hindering the domain wall propagation through the film plane, which makes to increase the coercivity of the antidots samples respect to the continuous thin films. Additionally, the magnetostatic dipolar interaction among the holes gives rise to a demagneti- zing field in the opposite direction to magnetization [27]. Furthermore, for the antidot samples we found an opposite thickness dependent behavior of $H_{c}$, as illustrated in Fig. 4, where it is clearly shown that coercivity decreases with increasing the Fe antidots thin film thickness.

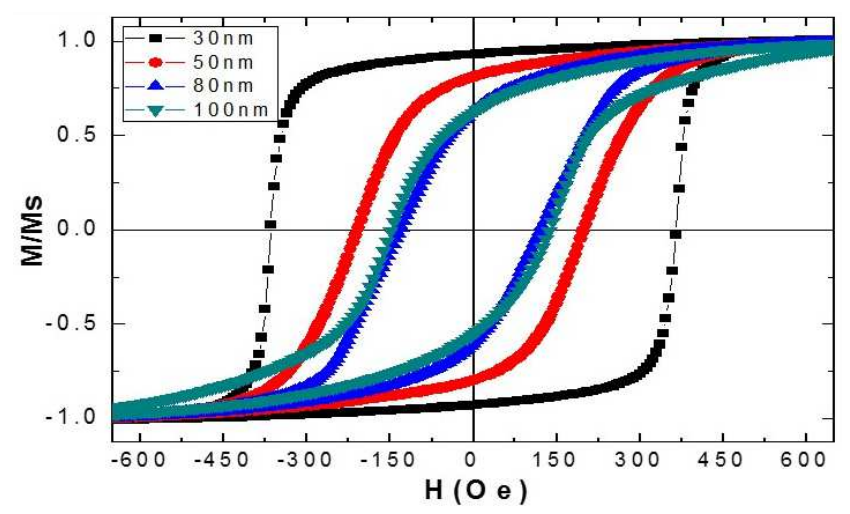

Fig. 4. Longitudinal Kerr hysteresis loops for Fe antidots thin films with different thicknesses $(30,50,80$, and $100 \mathrm{~nm})$.

With increasing the layer thickness, the geometry of thin films of antidots arrays changes and the magnetic behavior becomes also modified. The thickness increasing adds soft and hard magnetic behaviors due to the decrease of the holes size on the one hand, and the increase of the surface roughness of the film by the other side. The shape anisotropy of the thin film is strongly modified by the nanoholes and this enhances the uniaxial anisotropy in the film plane for the thinner samples (larger nanoholes sizes), due to the magnetostatic interactions among the accumulative magnetic poles of opposite sign at each side of the nanoholes that originates a pinning effect, which clearly decays when the nanoholes size diminishes with thickness layer increasing. The pinning effect ascribed to nanoholes dominates above the roughness surface anisotropy for antidots thin films with smaller layer thickness giving rise to higher coercivity values than in continuous thin films and a decrease of $H_{c}$ when the thickness of the $\mathrm{Fe}$ antidots thin films increases. Also, the antidots thin films with higher layer thickness tend to prefer the outof-plane magnetization direction [28]. Vázquez et al. [29] propose a phenomenological law which predicts that the coercivity follows a linear relationship with the thickness for the Ni antidots thin films, $H_{c} \approx(1 / t)(D-d)$, where $D$ is the interdistance between antidots centers, $d$ is the antidot diameter and $t$ - the layer thickness. In our case, the parameter $(D-d)$ remains constant, so $H_{c} \approx 1 / t$. This phenomenological law agrees well with our experimental data, where it can be seen that $H_{c}$ is inversely proportional to the $\mathrm{Fe}$ antidots thin film thickness.

\subsection{Cylindrical magnetic nanowires with modulated geometry}

The morphology, chemical composition, and crystalline structure of multisegmented nanowires with modulated diameter and composition has been studied by SEM, 
TEM and EDX techniques. Figure 5a displays a SEM cross-section view of diameter modulated Ni nanowires embedded into the nanoporous AAO template.
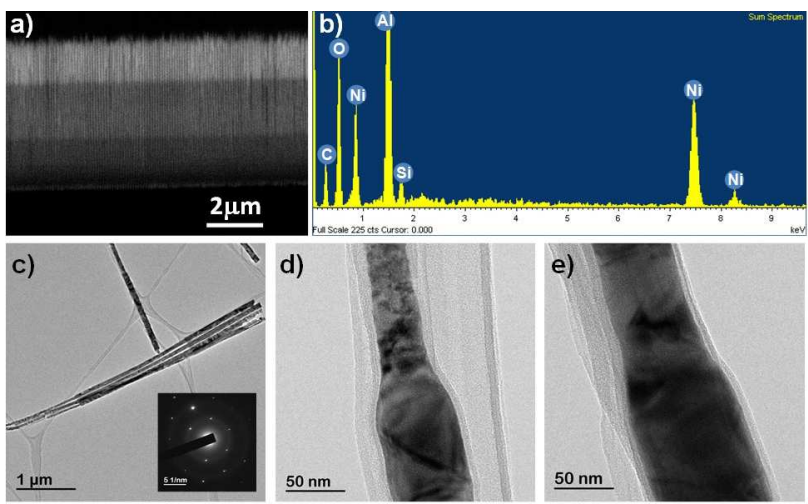

Fig. 5. (a) SEM cross-section view of sample G1, obtained with backscattered electron detector. (b) EDX spectrum of sample G1. (c) Low magnification TEM image of diameter modulated Ni nanowires from sample G2, after selective removal of the nanoporous AAO template. The inset shows SAED spectrum evidencing the crystallinity of the Ni nanowires. (d) High magnification TEM image of diameter modulation corresponding to segments 3-2 in sample G2. (e) High magnification TEM image of diameter modulation corresponding to segments $2-1$ in sample G2.

The compositional contrast, obtained by employing backscattered electron detector, allows distinguishing the different modulations in diameter. Three different segments are observed, with the narrower nanowires section at the bottom and the wider one at the top of the image. The EDX spectrum shown in Fig. 5b was recorded in the same region as the image in (a), and evidences the $\mathrm{Ni}$ composition of the nanowires, as well as the $\mathrm{Al}$ and $\mathrm{O}$ signals coming from the nanoporous alumina membrane, and the $\mathrm{Si}$ comes from the $\mathrm{SiO}_{2}$ functionalization by ALD. Image of Fig. $5 \mathrm{c}-\mathrm{e}$ shows TEM images of diameter modulated nanowires corresponding to sample G2, obtained after selective removal of the nanoporous AAO template by chemical etching. The low magnification image shown in Fig. 5c displays several Ni nanowires formed by three segments, each of which has different diameter size; while the high magnification ones in (d) and (e) show more clearly the different diameters between segments 3-2 and 2-1, respectively. From the above characterization, performed for samples G1-G4, the morphological parameters obtained are displayed in Table I.

As indicated by the larger variation in nanowire diameter modulation, the approach followed for the preparation of sample G2 is more efficient than that applied in the case of G1. Homogeneous nanowires, G3 and G4, display similar diameter to that of the narrow and intermediate segments in sample G2.

Hysteresis loops of Ni diameter modulated NWs grown in the nanoporous alumina templates were measured by VSM technique and are displayed in Fig. 6. The high aspect ratio Ni NWs with uniform diameter, or without
TABLE I

Lattice parameters: diameter $\Phi[\mathrm{nm}]$ and length $l[\mu \mathrm{m}]$ of samples G1 to G4.

\begin{tabular}{c|c|c|c|c|c|c}
\hline \hline & \multicolumn{2}{|c|}{ Seg. 1 } & \multicolumn{2}{c|}{ Seg. 2 } & \multicolumn{2}{c}{ Seg. 3 } \\
\cline { 2 - 7 } & $\Phi$ & $l$ & $\Phi$ & $l$ & $\Phi$ & $l$ \\
\hline G1 & 31 & 1.5 & 40 & 2 & 54 & 1.5 \\
G2 & 92 & 1.7 & 70 & 2.3 & 40 & 1.5 \\
G3 & 40 & 5 & & & & \\
G4 & 65 & 7 & & & &
\end{tabular}

diameter modulation, exhibit a sharp magnetization reversal, high coercive field and large squareness, $S$, for an applied magnetic field $(H)$ along the wire long axis (parallel $\|$ direction). The squareness is defined as the ratio between remanent $\left(M_{r}\right)$ and saturation $\left(M_{s}\right)$ magnetization. On the other hand, for $H$ perpendicular to the wire axis (perpendicular $\perp$ direction), hysteresis is reduced leading to lower values of $H_{c}$ and $S$. Therefore, the easy magnetization axis is parallel oriented to the wire axis, while the hard magnetization axis is perpendicularly oriented.

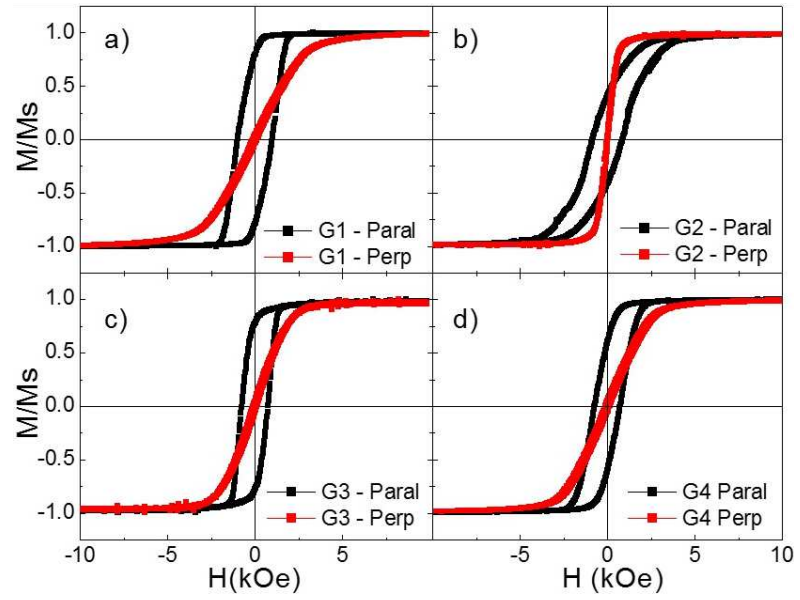

Fig. 6. Hysteresis loops of Ni NWs measured by VSM at room temperature for two applied directions of $H$ (\| and $\perp$ ). (a) Sample G1, (b) sample G2, (c) sample G3 and (d) sample G4.

For diameter modulated NWs, we observe similar magnetic properties having $H_{c}^{\|}>H_{c}^{\perp}$ and $S^{\|}>S^{\perp}$, but with different values. Small changes in the magnetic hysteresis curve of parallel magnetized loops can be clearly observed, attributed to changes of the magnetic susceptibility, meaning that the diameter modulation of magnetic nanowires is playing an important role in the magnetization reversal processes, acting as pinning centers for domain wall movements.

\section{Conclusions}

Bottom-up strategies based on template-assisted electrochemical methods allow synthesizing different kinds of nanostructured materials such as nanowires, nanotubes, 
or antidot thin films. Tuning the wire dimensions allows tailoring the physical properties of nanostructured materials and particularly the peculiar magnetic behavior exhibited by both, the single isolated nanowires and nanowires in the array. Diameter modulated nanowires can be considered as novel nanostructured materials that exhibit new interesting phenomena, since these geometrical modulations can act as pinning centers of the magnetic domain walls, which convert them in outstanding candidates for spintronic applications. Global and local magnetization behavior in Fe-based antidot thin films is strongly dependent of holes diameter and layer thickness. Tailored design of these novel nanostructured ferromagnetic materials would be of huge interest for applications in many research fields such as high-density magnetic data storage devices, magnetotransport phenomena, logical devices, magnonic crystals, or in magnetoplasmonics, among others.

\section{Acknowledgments}

Financial support under Spanish MINECO research projects No. MAT2013-48054-C2-2-R and MAT201676824-C3-3-R, together Principality of Asturias from FICyT project No. FC-15-GRUPIN14-085 are gratefully acknowledged. Common Services of Research from University of Oviedo SCT's, are also recognized.

\section{References}

[1] Z. Fan, R. Kapadia, P.W. Leu, X. Zhang, Y.L. Chueh, K. Takei, K. Yu, A. Jamshidi, A.A. Rathore, D.J. Ruebusch, M. Wu, A. Javey, Nano Lett. 10, 3823 (2010).

[2] Y.P. Ivanov, A. Chuvilin, S. Lopatin, J. Kosel, ACS Nano 10, 5326 (2016).

[3] T. Böhnert, V. Vega, A.-K. Michel, V.M. Prida, K. Nielsch, Appl. Phys. Lett. 103, 092407 (2013).

[4] H. Masuda, K. Fukuda, Science 268, 1466 (1995).

[5] V.M. Prida, J. García, L. Iglesias, V. Vega, D. Görlitz, K. Nielsch, E.D. Barriga-Castro, R. MendozaReséndez, A. Ponce, C. Luna, Nanoscale Res. Lett. 8, 263 (2013)

[6] V. Vega, T. Böhnert, S. Martens, M. Waleczek, J.M. Montero-Moreno, D. Görlitz, V.M. Prida, K. Nielsch, Nanotechnology 23, 465709 (2012).

[7] M.P. Proenca, K.J. Merazzo, L.G. Vivas, D.C. Leitao, C.T. Sousa, J. Ventura, J.P. Araujo, M. Vazquez, Nanotechnology 24, 425701 (2013).

[8] G.A. Badini Confalonieri, V. Vega, A. Ebbing, D. Mishra, P. Szary, V.M. Prida, O. Petracic, H. Zabel, Nanotechnology 22, 285608 (2011).

[9] C.C. Wang, A.O. Adeyeye, N. Singh, Nanotechnology 17,1629 (2006).

[10] F. Haering, U. Wiedwald, S. Nothelfer, B. Koslowski, P. Ziemann, L. Lechner, A. Wallucks, K. Lebecki, U. Nowak, J. Gräfe, E. Goering, G. Schütz, Nanotechnology 24, 465709 (2013).

[11] M. Knez, K. Nielsch, L. Niinistö, Adv. Mater. 19 3425 (2007).

[20] C. Marichy, M. Bechelany, N. Pinna, Adv. Mater. 24, 1017 (2012).
[12] W. Lee, R. Ji, U. Gösele, K. Nielsch, Nature Mater. 5, $741(2006)$

[13] V. Vega, J. García, J.M. Montero-Moreno, B. Hernando, J. Bachmann, V.M. Prida, K. Nielsch, ACS Appl. Mater. Interfaces 7, 28682 (2015).

[14] K. Pitzschel, J.M. Montero Moreno, J. Escrig, O. Albrecht, K. Nielsch, J. Bachmann, ACS Nano 3, 3463 (2009).

[15] J.M. Montero Moreno, M. Waleczek, S. Martens, R. Zierold, D. Görlitz, V. Vega Martínez, V.M. Prida K. Nielsch, Adv. Funct. Mater. 24, 1857 (2014).

[16] M.S. Salem, P. Sergelius, R.M. Corona, J. Escrig, D. Görlitz, K. Nielsch, Nanoscale 5, 3941 (2013).

[17] I. Minguez-Bacho, S. Rodriguez-López, M. Vázquez, M. Hernández-Vélez, K. Nielsch, Nanotechnology 25. 145301 (2014).

[18] V.M. Prida, J. García, B. Hernando, C. Bran, L.G. Vivas, M. Vázquez, in: Magnetic Nano- and Microwires, Design, Synthesis, Properties and Applications, Ed. M. Vázquez, Woodhead Publishing Series in Electronic and Optical Materials Elsevier, 2015, Ch. 2, p. 41 .

[19] A. Apolinário, P. Quitério, C.T. Sousa, M.P. Proença, J. Azevedo, M. Susano, S. Moraes, P. Lopes, J. Ventura, J.P. Araújo, J. Phys. Conf. Series 534, 012001 (2014).

[20] J. Bachmann, R. Zierold, Y.T. Chong, R. Hauert, C. Sturm, R. Schmidt-Grund, B. Rheinländer, U. Gösele, K. Nielsch, Angew. Chem. Int. Ed. 47, 6177 (2008).

[21] J. García, V. Vega, L. Iglesias, V.M. Prida, B. Hernando, E.D. Barriga-Castro, R. Mendoza-Reséndez, C. Luna, D. Görlitz, K. Nielsch, Phys. Status Solidi A 211, 1041 (2014).

[22] F. Béron, K.R. Pirota, V. Vega, V.M. Prida, A. Fernández, B. Hernando, M. Knobel, New J. Phys. 13. 013035 (2011).

[23] K.R. Pirota, P. Prieto, A.M.J. Neto, J.M. Sanz, M. Knobel, M. Vazquez, J. Magn. Magn. Mater. 320, e235 (2008).

[24] H. Jia, X. Wang, S.-P. Pang, W.-T. Zheng, B.H. Long, B. Li, Trans. Nonferrous Met. Soc. China 19, $353(2009)$.

[25] D.C. Leitao, J. Ventura, J.M. Teixeira, C.T. Sousa, S. Pinto, J.B. Sousa, J.M. Michalik, J.M. de Teresa, M. Váquez, J.P. Araujo, J. Phys. Condens. Matter. 25, 6 (2013).

[26] K.J. Merazzo, C. Castán-Guerrero, J. HerreroAlbillos, F. Kronast, F. Bartolomé, J. Bartolomé, J. Sesé, R.P. del Real, L.M. García, M. Vázquez, Phys. Rev. B 85, 104427 (2012).

[27] Y.-P. Zhao, R.M. Gamache, G.-C. Wang, T.-M. Lu, G. Palasantzas, J.Th.M. De Hosson, J. Appl. Phys. 89, 1325 (2001).

[28] M. Jaafar, D. Navas, A. Asenjo, M. Vázquez, M. Hernández-Vélez, J.M. García-Martín, J. Appl. Phys. 101, 09F513 (2007).

[29] M. Vazquez, K.R. Pirota, D. Navas, A. Asenjo, M. Hernandez-Velez, P. Prieto, J.M. Sanz, J. Magn. Magn. Mater. 320, 1978 (2008). 